\title{
Production of Relativistic Electron Bunch with Tunable Current Distribution
}

\author{
P. Piot*, Y.-E Sun ${ }^{\dagger}$ and M. Rihaoui** \\ ${ }^{*}$ Department of Physics and Northern Illinois Center for Accelerator \& Detector Development, \\ Northern Illinois University, IL 60115 DeKalb, USA \\ and Accelerator Physics Center, Fermi National Accelerator Laboratory, Batavia IL 60510, USA \\ ${ }^{\dagger}$ Accelerator Physics Center, Fermi National Accelerator Laboratory, Batavia IL 60510, USA \\ ** Department of Physics and Northern Illinois Center for Accelerator \& Detector Development, \\ Northern Illinois University, IL 60115 DeKalb, USA
}

\begin{abstract}
We propose a novel method for tailoring the current distribution of relativistic electron bunches. The technique relies on a recently proposed transverse-to-longitudinal phase space exchange. The bunch is transversely shaped and the phase space exchange mechanism converts this transverse profile into a current profile. The technique provides a tool for generating arbitrary current profiles in a tunable fashion. We demonstrate, via computer simulations, the method and its application to tailor specific current profiles such as, e.g., linearly ramped profiles and train of femtosecond micro-bunches that have application in plasma and dielectric wakefield accelerators.
\end{abstract}

Keywords: beams, phase space manipulation, beam dynamics

PACS: $29.20 . \mathrm{Ej}, 29.25 .-\mathrm{t}, 29.27 .-\mathrm{a}, 29.27 . \mathrm{Bd}$

\section{INTRODUCTION}

Modern applications of accelerators often call for phase space manipulation within one or two degree-of-freedom to tailor the phase space distribution for a specific application. Often such a phase space matching is done in a root-mean-square approach. Novel applications of charged particle beams call for more precise phase space control. It is in particular sometime desired to produce electron bunch with a well-defined current profile. Such a need is exemplified in accelerator-based light sources based on the freeelectron laser (FEL) principle and using the so-called high gain harmonic generation (HGHG) that requires very uniform current profile. The production of comb bunches, i.e. a bunch consisting of a microbunches train, could also open the path toward compact light source operating in the super-radiant regime at a wavelength comparable or larger than the typical density modulation. Another class of applications concerns novel beam-driven acceleration mechanisms, e.g. those based on plasma or dielectric wakefield acceleraton. In these applications, linearly ramped current or trapezoidal profiles have been shown to significantly increase the transformer ratio - the energy gain of the accelerated bunch over the energy lost by the driving bunch. Similarly the performance of dielectric wakefield accelerators, capable of $\mathrm{GeV} / \mathrm{m}$ at sub-millimeter wavelength, could be significantly enhanced if driven by a linearly ramped comb bunch with microbunches separated by the desired excitation frequency thereby enabling multi-GeV table-top accelerators driven by medium-to-low energy accelerator drivers. 
To address these needs several groups have investigated method for generating various type of current profiles. Linearly ramped current profile can in principle be produced by imparting nonlinear distortions in the longitudinal phase space. An example using a nonlinear optical lattice was recently demonstrated [1]. The generation of comb bunches by shaping the photocathode drive laser of a photoemission electron source were explored via numerical simulations $[2,3]$. An alternative method to produce comb bunches using an interceptive mask located in a dispersive section was experimentally demonstrated [4]. Finally the tailoring of the current profile to enhance the operation of a FEL operating in the HGHG mode by an ad hoc shaping of the photocathode drive laser used to produce the electron beam was discussed in Reference [5]. Each of these methods has its limitation: the methods based on shaping the photocathode drive laser distribution are prone to the space-charge effects which are prominent at low energy and tend to wash out the impressed density modulations. Other techniques such as those based on nonlinear transformations have a limited tunability.

\section{CONCEPT}

In this Paper we present a general technique for tailoring the current profile to follow any distribution. The method is illustrated in Figure 1: an incoming electron bunch is transversely intercepted by a mask with transmission function $T(x, y)$. The mask is shaped to result in certain shape of the horizontal beam projection. A transverseto-longitudinal emittance exchanger is used to map this horizontal projection onto the longitudinal (temporal) direction.

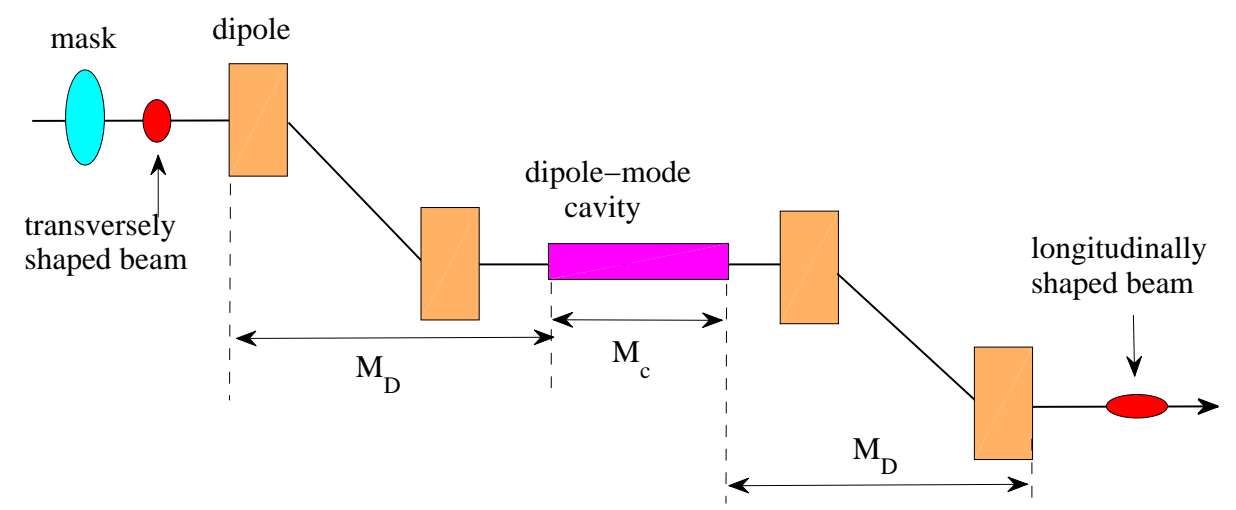

FIGURE 1. Overview of the proposed technique to produced relativistic electron bunch with arbtrary current profile. The emittance exchanger beamline is composed of four dipoles (shown as orange rectangles) and an horizontally-deflecting cavity (magenta rectangle).

The backbone of our proposed scheme is a transverse-to-longitudinal phase space exchanger which was first proposed to achieve ultra-low beta function in B-factory [6] and later explored as a possible alternative for mitigating potential microbunching instability [7] or improving the performance of single-pass FELs [8]. Considering the phase space exchange between the horizontal $\left(x, x^{\prime}\right)$ and longitudinal $(z, \delta)$ phase spaces, such exchanger is composed of an horizontally deflecting cavity, operating on the $\mathrm{TM}_{110}$ mode, flanked by two horizontally dispersive sections. Considering single-particle dy- 
namics in the four dimensional phase space $\left(x, x^{\prime}, z, \delta\right)$, an initial electron with coordinate $\overline{\mathbf{X}}_{\mathbf{0}} \equiv\left(x_{0}, x_{0}^{\prime}, z_{0}, \delta_{0}\right)$ is mapped to its final coordinate $\overline{\mathbf{X}} \equiv\left(x, x^{\prime}, z, \delta\right)$ accordingly to $\overline{\mathbf{X}}=R \overline{\mathbf{X}}_{\mathbf{0}}$ wherein $R$ is a $4 \times 4$ transport matrix having a block anti-diagonal form [8, 9].

In order to illustrate how this emittance exchange mechanism could be used to tailor the longitudinal distribution of an electron bunch we henceforth consider the initial trace space distribution associated to the beam to follow a six-dimensional Gaussian distribution of the form

$$
\Phi_{0}\left(\mathbf{X}_{\mathbf{0}}\right)=\frac{n_{0}}{(2 \pi)^{2} \varepsilon_{x, 0} \varepsilon_{y, 0} \varepsilon_{z, 0}} \exp \left[-\frac{1}{2} \mathbf{X}_{\mathbf{0}} \Sigma_{0}^{-1} \widetilde{\mathbf{X}_{\mathbf{0}}}\right]
$$

where $\mathbf{X}_{0} \equiv\left(x_{0}, x_{0}^{\prime}, y_{0}, y_{0}^{\prime}, z_{0}, \delta_{0}\right)$, and $\Sigma_{0} \equiv\left\langle\mathbf{X}_{\mathbf{0}} \widetilde{\mathbf{X}}_{\mathbf{0}}\right\rangle$ is the six-dimensional covariance matrix associated to the beam (the tilde stands for the transposition operation). We further assume the initial covariance to be uncoupled i.e. to have the form $\Sigma_{0}=$ $\operatorname{diag}\left(\Sigma_{x, 0}, \Sigma_{y, 0}, \Sigma_{z, 0}\right)$ where

$$
\Sigma_{i, 0} \equiv \varepsilon_{i, 0}\left[\begin{array}{cc}
\beta_{i, 0} & -\alpha_{i, 0} \\
-\alpha_{i, 0} & \gamma_{i, 0}
\end{array}\right]
$$

where the index $i=x, y, z$ stands for the considered degree of freedom and $\alpha_{i, 0}, \beta_{i, 0}$ and $\gamma_{i, 0} \equiv\left(1+\alpha_{i, 0}^{2}\right) / \beta_{i, 0}$ are the usual Courant-Snyder parameters.

Here we consider the initial distribution function can be transversely shaped via a mask with transmission $T(x, y)$. We write the mask boundaries to be of the form $y^{+}(x)$ and $y^{-}(x)$ for respectively the upper $(y>0)$ and lower $(y<0)$ domain of the $(x, y)$ plane. Upon integration over $y$ and $y^{\prime}$, the four dimensional phase space distribution at the exchanger entrance is

$$
\begin{aligned}
\Phi_{0}^{+}\left(\overline{\mathbf{X}}_{\mathbf{0}}\right) & =\Phi_{0}^{-}\left(\overline{\mathbf{X}}_{\mathbf{0}} ; 0\right) \int_{y_{0}^{-}(x)}^{y_{0}^{+}(x)} \frac{d y_{0}}{\sqrt{2 \pi} \sigma_{y, 0}} \times \exp \left(-\frac{y_{0}^{2}}{2 \beta_{y, 0} \varepsilon_{y, 0}}\right) T\left(x_{0}, y_{0}\right) \\
& \equiv \Phi_{0}^{-}\left(\overline{\mathbf{X}}_{\mathbf{0}} ; 0\right) \tau\left(R^{-1} \overline{\mathbf{X}}_{\mathbf{0}}\right) .
\end{aligned}
$$

Downstream of the exchanger the final distribution is

$$
\Phi(\overline{\mathbf{X}} ; \mathbf{s})=\Phi\left(R^{-1} \overline{\mathbf{X}}_{\mathbf{0}} ; 0\right) \tau\left(R^{-1} \overline{\mathbf{X}}_{\mathbf{0}}\right)
$$

and the longitudinal phase space is given by

$$
\begin{aligned}
\Phi(z, \delta)= & \frac{n_{0}}{\sqrt{2 \pi} \varepsilon_{z, 0}} \exp \left(-\frac{z^{2}}{2 \beta_{z} \varepsilon_{x, 0}}\right) \\
& \times \exp \left[-\frac{\beta_{z}}{2 \varepsilon_{x, 0}}\left(\delta-\frac{\alpha_{z}}{\beta_{z}} z\right)^{2}\right] \times \tau\left(N_{15} z+N_{16} \delta\right),
\end{aligned}
$$

where $N \equiv R^{-1}$. The latter distribution describes a two-dmensional Gaussian distribution in $(z, \delta)$ modulated by the function $\tau$. Integrating over $\delta$ yields the longitudinal profile 
downstream of the exchanger:

$$
P(z)=\frac{n_{0}}{2 \pi \varepsilon_{x, 0}} \exp \left(-\frac{z^{2}}{2 \beta_{z} \varepsilon_{x, 0}}\right) \Theta(z),
$$

with $\Theta(z)=\int_{-\infty}^{+\infty} \exp \left[-\frac{\beta_{z}}{2 \varepsilon_{x, 0}}\left(\delta-\frac{\alpha_{z}}{\beta_{z}} z\right)^{2}\right] \tau\left(N_{15} z+N_{16} \delta\right) d \delta$. If the mask has no ydependence $\left[y^{+}(x) \rightarrow+\infty\right.$ and $\left.y^{-}(x) \rightarrow-\infty\right], \tau$ is a simple function of $x$.

We now consider the case of $\tau\left(x_{0}\right)=\sum_{i=1}^{N} \delta\left(x-x_{0}^{i}\right)$ where $\delta()$ is the Dirac function. This can be achieved, e.g., by intercepting the incoming beam with a series of vertical slits with centers located at $x_{0}^{i}$. In such a case the longitudinal phase space is described by a two-dmensional Gaussian distribution with Courant-Snyder parameters $\left(\alpha_{x}, \beta_{z}\right)$, emittance $\varepsilon_{x, 0}$, which is non-vanishing only at locations described by the family of lines with equation $\delta(z)^{i}=x_{0}^{i}+N_{15} / N_{16} z$. Although a projection along the longitudinal axis does not generally provide a density-modulated electron bunch, the local correlations in the longitudinal phase space can be exploited using a dispersive section located downstream of the exchanger to further bunch the microbunches. To do so the longitudinal dispersion $R_{56} \equiv \int \eta(s) / \rho(s) d s$ of the dispersive section should matched to the chirp of the microbunches, i.e. $R_{56}=-N_{15} / N_{16}$.

\section{NUMERICAL MODELING}

To explore the technique we consider an incoming beam with Gaussian distribution traveling though a shaper section, the emittance exchanger and a bunch compressor located downstream. In this paper we only concentrate on the single-particle dynamic calculation. The beamline geometric parameters are gathered in Table 1 and our simulations are based on the numerical model elaborated in Reference [10]. In a first example we

TABLE 1. Geometric parameters associated to the beamline exchange considered in this paper.

\begin{tabular}{lccc}
\hline parameter & symbol & value & unit \\
\hline Bending angle & $\alpha$ & 22.5 & $\mathrm{deg}$ \\
Effective bend length & $L_{b}$ & 0.20 & $\mathrm{~m}$ \\
Distance between bends & $D$ & 0.75 & $\mathrm{~m}$ \\
Dogleg dispersion & $\eta$ & 0.25 & $\mathrm{~m}$ \\
\hline
\end{tabular}

explore the case of a $15 \mathrm{MeV}$ electron bunch passing through a series of vertical slits upstream of the exchanger. The slits are $100 \mu \mathrm{m}$ wide and separated by $200 \mu \mathrm{m}$. The corresponding longitudinal phase space downstream of the emittance exchanger is shown in Fig. 2. As predicted by our analytical considerations the longitudinal phase space is fragmented in a series of parallel "stripes" with same $z-\delta$ correlations. In this example the microbunches can be further compressed using a magnetic bunch compressor (with $R_{56}$ sign opposites to a standard four-bend chicane) to yield a train of microbunches with duration below $\sim 50 \mathrm{fs}(\mathrm{rms})$.

In Fig. 3 we present an example of generation of linear-ramped bunches for an incoming beam with parameters similar to those previously mentioned. Producing arbi- 

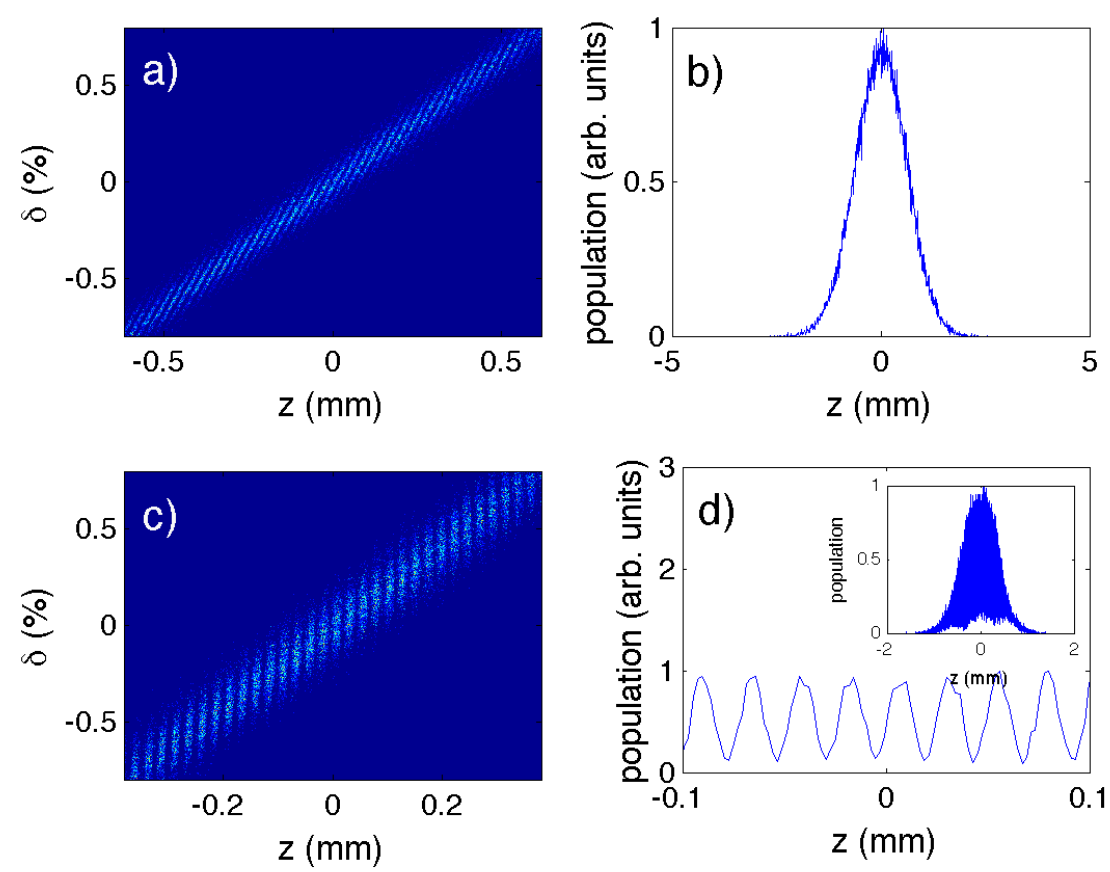

FIGURE 2. Production of a train of sub $100 \mathrm{fs}$ bunches. The beam upstream of the emittance exchanger is passed through a set of vertical slits $(0.1 \mathrm{~mm}$ wide $)$ separated by $0.2 \mathrm{~mm}$. The longitudinal phase spaces and associated longitudinal projections are shown downstream of the emittance exchanger beamline [(a) and (b)], and downstream of a bunch compressor with $\left|R_{56}\right| \simeq 6 \mathrm{~cm} \mathrm{[(c)} \mathrm{and} \mathrm{(d)].} \mathrm{Plot} \mathrm{(d)} \mathrm{is} \mathrm{a} \mathrm{zoomed} \mathrm{in}$ of the charge density for $-0.1<z<0.1 \mathrm{~mm}$ - the inset shows the total charge distribution. In these plots $z>0$ corresponds to the bunch head.

trary profiles relies on the transverse shaping of the beam spot in the $(x, y)$ plane to provide the desired horizontal projection (which will eventually be mapped on the temporal axis downstream of the exchanger). Intercepting the beam with a mask having $y$-dependent aperture could in principle generate any distribution provided $y(x)$ is accordingly tailored. It could however result in significant beam loss. Shaping the beam in the transverse $(x, y)$ might also be accomplished non-interceptively, e.g., via nonlinear (or coupling) optics, or by generating a multi-beam with a predefined pattern from a photocathode electron source.

\section{SUMMARY \& POSSIBLE APPLICATIONS}

In summary we presented a general concept for potentially generating relativistic electron beam with arbitrary current profiles. Nominally the technique is interceptive thereby potentially resulting in significant charge loss. It can be straightforwardly implemented at low energies $(<50 \mathrm{MeV})$ and be used to "feed-forward" the appropriate current profile, e.g., to compensate for additional longitudinal phase space manipulations (bunch compressor) or deleterious effects (wakefields) that may happen in the downstream 

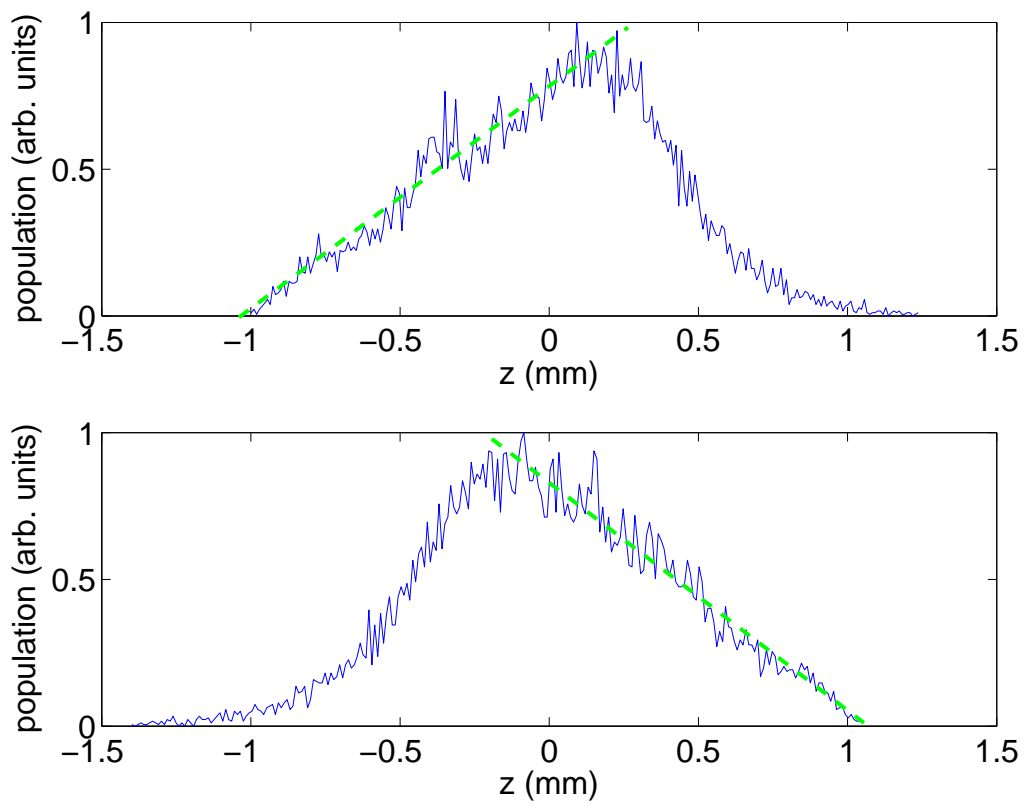

FIGURE 3. Production of a linearly-ramped bunches. The beam was passed through an aperture with horizontally-dependent height. Beam with descending (top) or rising (bottom) linear ramp were produced.

beamline. The concept seems promising but its application to particular problem must be considered in details especially since the technique exchange the horizontal and transverse emittances.

\section{ACKNOWLEDGMENTS}

The work of P. P. and M. R. was supported by the US Department of Energy under Contract No. DE-FG02-08ER41532 with Northern Illinois University. The work of Y.-E S. was supported by the Fermi Research Alliance, LLC under Contract No. DE-AC0207CH11359 with the U.S. Department of Energy.

\section{REFERENCES}

1. R. J. England, J. B. Rosenzweig, and G. Travish, Phys. Rev. Lett. 100, 214802 (2008).

2. M. Bolosco, et al., Nucl. Instr. Meth. A 577, 409 (2007).

3. Y. Li, and K.-J Kim, Appl. Phys. Lett 92, 014101 (2008).

4. P. Muggli, et al., Phys. Rev. Lett. 101, 054801 (2008).

5. M. Cornacchia, S. Di Mitri, G. Penco, and A. Zholents, Phys. Rev. ST Accel. Beams 9, 120701 (2006).

6. Y. Orlov et al., Proc. of PAC91, San Francisco, 2838 (1991).

7. M. Cornachia, and P. Emma, Phys. Rev. ST Accel. Beams 6030702 (2003).

8. P. Emma, Z. Huang, K.-J. Kim, and P. Piot, Phys. Rev. ST Accel. Beams 9, 100702 (2006).

9. Y.-E Sun, et al, Proc. of the PAC07, 3441 (2007).

10. M. Rihaoui, et al., Proc. 13th Advanced Accelerator Concepts Workshop, Santa Cruz, CA (2008). 